\title{
Cardiac Amyloidosis and Aortic Stenosis: When to Consider it and How to Treat it?
}

\author{
Marcelo Kirschbaum, Aristóteles Conte Alencar Neto, Vitor Emer de Egypto Rosa, Félix José Alvarez Ramires, Alexandre \\ Antonio Cunha Abizaid, Fábio Sandoli de Brito Junior Flávio Tarasoutchi, Roberto Kalil Filho, Fábio Fernandes ${ }^{(1)}$ \\ Instituto do Coração Incor-HC FMUSP, São Paulo, SP - Brazil
}

\begin{abstract}
Aortic stenosis is the most prevalent valve disease in clinical practice. The wild form of transthyretin amyloidosis has an increased incidence in elderly individuals, usually over 70 years of age. Amyloidosis and aortic stenosis are 2 diseases that affect similar populations; therefore, they may coexist in the same patient.

Patients with amyloidosis and aortic stenosis have disproportionately higher serum levels of troponin and NTproBNP compared to patients with pure severe aortic stenosis in addition to greater ventricular hypertrophy. Therapeutic decisions for these patients must be individualized and promptly discussed by the heart team. In this article, we discuss this association and possible valve therapies, in addition to the possibility of specific treatment for amyloidosis.
\end{abstract}

\section{Clinical case}

J.P.S., a 73-year-old, male patient who was born and raised in Bahia, had prior diagnosis of systemic arterial hypertension, benign prostatic hyperplasia, and bilateral glaucoma. Regarding family history, his father had died due to Chagas disease, and his mother had died of an acute myocardial infarction. He was using losartan 50 mg, acetylsalicylic acid $100 \mathrm{mg}$, and finasteride/doxazosin.

He sought outpatient care, reporting that, 2 months prior, he had started to experience progressive dyspnea upon moderate exertion, orthopnea, and tachycardic palpitations. On physical examination, the presence of a rough midsystolic ejection murmur $3+/ 6$ radiating to the carotids and a blowing protodiastolic murmur $3+/ 6$, both in aortic focus, stood out. There were no signs of significant edema or turgescent jugular veins. At that time, the syndromic diagnosis of heart failure was suggested, with anatomical and etiological diagnosis of double degenerative aortic lesion. Furosemide $40 \mathrm{mg}$ was prescribed and additional tests were requested.

He returned 2 months later with dyspnea upon minimal exertion, as well as the following additional exams:

\section{Keywords}

Aortic Stenosis; Amyloidosis; Heart failure; preserved ejection fraction

Mailing Address: Fábio Fernandes•

Cardiomyopathies Group. Av Dr Enéas de Carvalho Aguiar, 44.

Postal Code: 05403-000, Cerqueira César, SP - Brazil.

E-mail: fabio.fernandes@incor.usp.br

Manuscript received September 19, 2021, revised manuscript September 30 2021, accepted September 30, 2021.

DOI: https://doi.org/10.36660/abchf.20210027
- 12-lead electrocardiogram: sinus bradycardia and left chamber overload.

- Two-dimensional echocardiography: important fibrocalcification in the aortic valve, with reduced valve mobility, maximum jet velocity of $4.9 \mathrm{~m} / \mathrm{s}$, and mean systolic gradient of $50 \mathrm{mmHg}$ (Velocities $>4 \mathrm{~m} / \mathrm{s}$ and mean gradient $>40 \mathrm{mmHg}$ indicate important stenosis); mild to moderate aortic regurgitation and pulmonary artery systolic pressure estimated at $50 \mathrm{mmHg}$. Biventricular systolic function was preserved; he had grade I diastolic dysfunction and moderate atrial enlargement.

Based on this new information, aortic valve replacement with a biological prosthesis was indicated, and preoperative tests were requested. Coronary cineangiography did not demonstrate any obstructive lesions. Euroscore II was 1.45\%.

Three years after the first evaluation, the patient was submitted to aortic valve replacement with a $23 \mathrm{~mm}$ biological prosthesis, and myectomy was performed due to the intraoperative finding of subvalvular aortic membrane. The surgery occurred without complications.

During anatomopathological study of the myectomy, morphological and immunohistochemical alterations consistent with transthyretin cardiac amyloidosis were observed. Subsequently, monoclocal gammopathy was ruled out by means of serum and urinary immunofixation and kappa and lambda immunoglobulin assay. The patient underwent pyrophosphate scintigraphy, demonstrating Perugini grade III uptake (Figure 1), and genetic testing showed no variants that would justify the clinical condition. This case illustrates the concomitant association of wild-type transthyretin systemic amyloidosis (ATTRwt) with cardiac involvement and aortic stenosis, with difficult clinical diagnosis.

\section{Introduction}

Aortic stenosis is the most prevalent valve disease in clinical practice, with an estimated prevalence of $0.2 \%$ in people over 50 years of age and a prevalence of up to $10 \%$ in octogenarians. This condition is characterized by aortic valve degeneration and calcification causing a fixed obstruction in the left ventricular outflow tract. Due to the pressure overload caused by aortic stenosis, the heart is affected by concentric remodeling, characterized by left ventricular wall hypertrophy and increased left ventricular filling pressures. ${ }^{1}$

Amyloidosis is characterized by the deposition of amyloid fibrils in the extracellular space of several organs, such as the heart, peripheral nerves, and gastrointestinal tract. The 2 most common types of amyloidosis are those caused by monoclonal proteins $(\mathrm{AL})$ and those caused by hepatic transthyretin (TTR) 
proteins. The TTR form can be further divided into 2 types: hereditary, when there is a known genetic mutation that causes the disease (ATTRh) and the wild type (ATTRwt, previously known as senile amyloidosis) (2). ATTR amyloidosis was considered a rare cause of heart failure, but it was perhaps only underdiagnosed. ATTRwt has an increased incidence in elderly individuals, usually over 70 years of age. On the other hand, necropsy studies have shown a prevalence of up to $25 \%$ of amyloidosis in patients over 80 years of age.,.$^{2,3}$

ATTR and aortic stenosis are 2 diseases that affect similar populations; therefore, they may coexist in the same patient.

The prevalence of concomitant amyloidosis and aortic stenosis ranges from $4 \%$ to $16 \%$. The smallest sample occurred in patients who underwent aortic valve replacement surgery and biopsy during the procedure. ${ }^{4}$ In a systematic study in 2 centers from the United States that used myocardial scintigraphy with technetium in all patients with aortic stenosis who underwent transcatheter aortic valve implantation (TAVI), a prevalence of $13 \%$ was described. ${ }^{5}$ On the other hand, the National Amyloidosis Centre in the United Kingdom, in a cohort of 1240 patients with amyloidosis, performed echocardiography and found severe aortic stenosis in $1.8 \%{ }^{6}$

In the vast majority of cases where aortic stenosis and amyloidosis coexist, the amyloid form is, generally, ATTRwt, and fewer than $5 \%$ of cases are the AL form. ${ }^{7}$

Given that both diseases are prevalent in the elderly population and that they cause similar structural changes in the heart (disproportionate hypertrophy, increased filling pressures, early change in global longitudinal strain), it is difficult to differentiate between patients with aortic stenosis who have amyloidosis and those who do not.

Establishing diagnosis of amyloidosis requires a high degree of clinical suspicion. Correct and accurate diagnosis is necessary, because conventional therapy for heart failure is not always well tolerated in cardiac amyloidosis. Prognosis is different from other etiologies of heart failure, and evolution and clinical management are different from other cardiomyopathies with a hypertrophic phenotype. Furthermore, the current specific therapeutic possibilities can modify the natural history of the disease.

In some studies, ${ }^{8}$ average time from onset of symptoms to establishment of diagnosis is up to 4 years in ATTRwt. In the case reported, it is possible to observe the patient's long journey, 36 months, to receive definitive diagnosis. In this context, imaging exams, especially echocardiography, scintigraphy, and cardiac magnetic resonance, have increasingly contributed to recognition of cardiac amyloidosis. The most important aspect, however, is to consider the disease.

\section{Diagnosis}

There are some red flags that have been described in the literature, which may help differentiate patients with aortic stenosis who may have concomitant amyloidosis. Patients with amyloidosis and aortic stenosis have disproportionately higher serum levels of troponin and NT-proBNP compared to patients with pure severe aortic stenosis; they may have greater ventricular thickness, and they may have more typical changes in the strain echocardiogram, such as the apical sparing pattern. ${ }^{9,10}$ We must take into account, however, that patients with severe aortic stenosis typically show early changes in global longitudinal strain; this fact that can interfere with the appearance of apical sparing caused by amyloidosis. In a study that evaluated the echocardiography pattern of mean $S$ wave velocity, measurement $<6 \mathrm{~cm} / \mathrm{s}$ was considered the best independent predictor of amyloidotic cardiomyopathy, with $100 \%$ sensitivity. ${ }^{9}$

Another condition that may be related to cardiac amyloidosis is low-flow, low-gradient aortic stenosis, both in cases of reduced left ventricular ejection fraction (LVEF), where the patient's mean systolic gradient in the aortic valve is $>40 \mathrm{mmHg}$ but valve area is $\leq 1.0 \mathrm{~cm}^{2}$, and in cases of paradoxical aortic stenosis, where the patient has small cavities due to extreme concentric remodeling, generating low-flow, low-gradient aortic stenosis with normal ejection fraction, mean systolic gradient $<40 \mathrm{mmHg}$, valve area $\leq$ $1.0 \mathrm{~cm}^{2}$ and normal LVEF.

Although they have been described in the literature, no study has found red flags that were adequate for giving rise to suspicion of cardiac amyloidosis in patients with aortic stenosis, given that cardiac remodeling caused by both diseases has similar, confounding characteristics.

A study by Sperry et al. outlining factors that demonstrated this association drew attention to the presence of carpal tunnel syndrome and a mismatch between low voltage and ventricular mass on electrocardiogram. ${ }^{11}$ Nitsche et al. developed the RAISE score, which takes ventricular remodeling, age, troponin, systemic involvement, and electrocardiographic changes into account; it showed good specificity and sensitivity for suspected amyloidosis in patients with severe aortic stenosis. ${ }^{12}$ A score greater than or equal to 2 had a sensitivity of $84 \%$, and a score greater than or equal to 3 had a specificity of $94 \%$ for detection of cardiac amyloidosis. Thus, a score greater than or equal to 2 is already a good milestone to begin confirmatory investigation of cardiac amyloidosis, but this score requires further studies for validation (Table 1).

Patients who undergo TAVI also undergo tomography for preoperative evaluation; currently, this method allows assessment of the extravascular space, which is enlarged in amyloidosis. ${ }^{13}$ Accordingly, this could be yet another exam to aid in screening these 2 conditions.

\section{Treatment}

Both diseases have poor prognosis if they are not identified and treated. Treatment of aortic stenosis consists of aortic valve intervention to reverse the structural disease. In elderly patients, TAVI has been shown to be the procedure of choice in all patient profiles, due to its safety and efficacy. ${ }^{14,15}$

The central question would be whether symptoms are due to a hemodynamic valve problem or to the myocardial disease that results from amyloid deposits. In relation to this, several studies have evaluated the effectiveness of treatment of aortic stenosis in patients with TTR cardiac amyloidosis. 
Review Article

Table 1 - Table 1 Multiple parameters applied by Nitsche et al. (2021)

\begin{tabular}{lr}
\hline Age $>85$ years & 1 point \\
\hline Carpal tunnel syndrome & 3 points \\
\hline Right bundle branch block & 2 points \\
\hline Sokolow-Lyon index $<1.9 \mathrm{mV}$ & 1 point \\
\hline High sensitivity troponin $>29 \mathrm{ng} / \mathrm{mL}$ & 1 point \\
\hline E/A $>1.4$ & 1 point \\
\hline
\end{tabular}

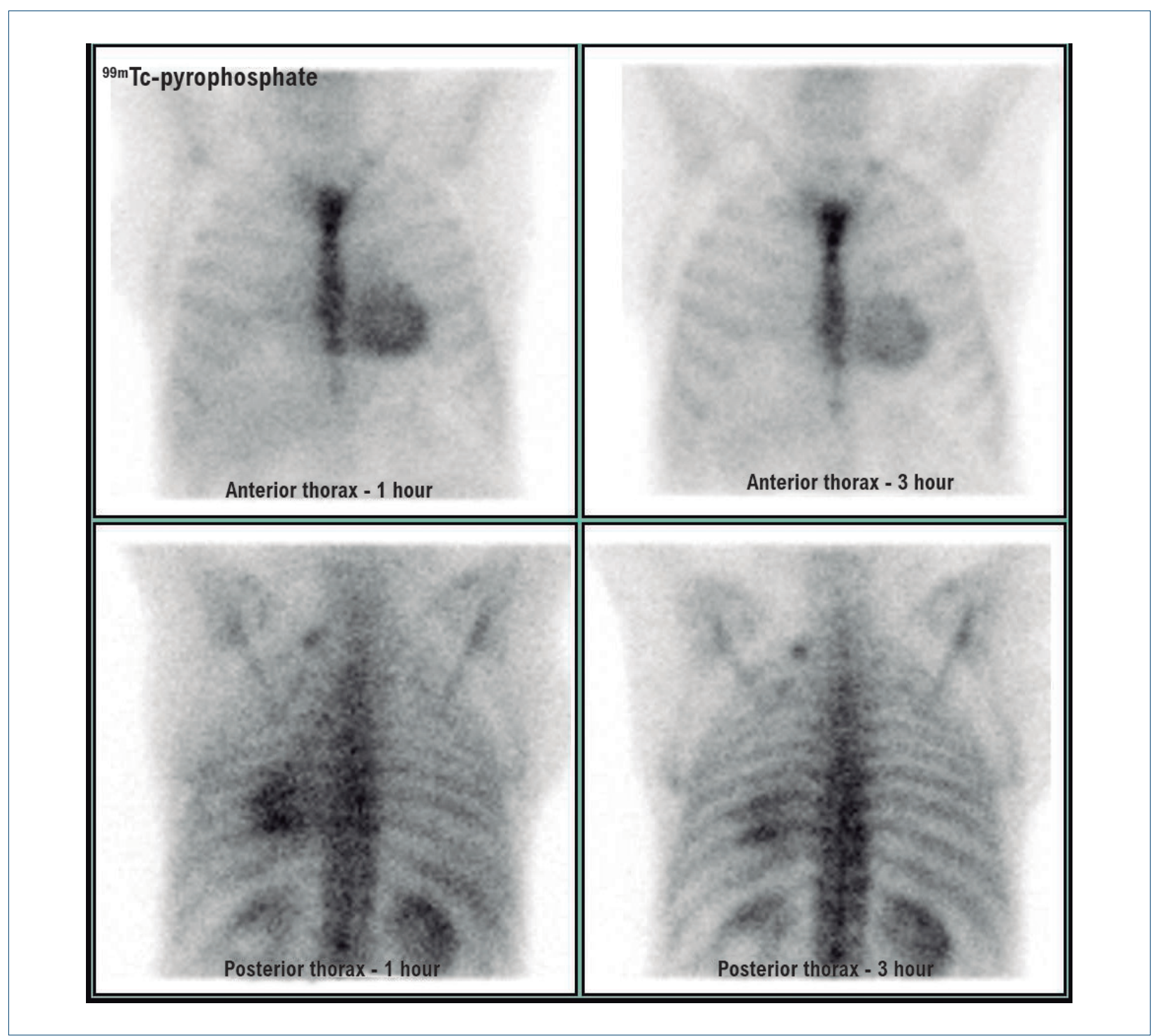

Figure 1 - Technetium pyrophosphate bone scintigraphy showing Perugini grade III uptake, 1 and 3 hours

In contrast to the initial study by Cavalcante et al., which provided evidence of higher mortality in patients with amyloidosis who underwent TAVI in comparison with patients with pure aortic stenosis, ${ }^{16}$ the most recently published studies that have used pyrophosphate scintigraphy to screen for amyloidosis in patients with aortic stenosis showed evidence that the patients who were submitted to aortic valve intervention benefit from the procedure, regardless of cardiac amyloidosis. ${ }^{12,16}$

With respect to defining which valve intervention to choose for patients with cardiac amyloidosis, Treibel et al. identified amyloid protein deposits in $6 \%$ of patients who underwent endomyocardial biopsy during aortic valve replacement surgery, and the presence of amyloidosis was a predictor of 
negative outcomes in the patients evaluated. ${ }^{4}$ Rosenblum et al. identified $13 \%$ of cases of cardiac amyloidosis in patients who underwent TAVI, and, in this population, the presence of amyloidosis was not identified as an outcome predictor. ${ }^{5}$ In spite of all these studies, we still do not have any randomized clinical trials that demonstrate the best intervention for aortic stenosis in patients with cardiac amyloidosis.

As amyloidosis induces a fragile state in patients, it predisposes them to arrhythmias and atrioventricular blocks, and TAVI has been shown to be safe in this population. We consider that, when possible, TAVI should be the procedure of choice for elderly patients with aortic stenosis and concomitant amyloidosis. Specific treatment for amyloid heart disease with transthyretin stabilizing drugs may impact the clinical course of patients.

The use of tafamidis was tested in the Tafamidis Treatment for Patients with Transthyretin Amyloid Cardiomyopathy (ATTR-ACT) study. This was a multicenter clinical randomized, placebo-controlled trial, involving 441 patients with cardiac amyloidosis, 264 of whom received the drug in doses of 20 $\mathrm{mg}$ or $80 \mathrm{mg}$ daily. The main results showed that the use of tafamidis was associated with a $30 \%$ reduction in the primary outcome of all-cause mortality $(\mathrm{RR}=0.70$ [95\% Cl: 0.51 0.96]); moreover, it reduced cardiovascular hospitalizations by $32 \%(\mathrm{RR}=0.68[95 \% \mathrm{Cl}: 0.56-0.81])$ and reduced worsening of functional capacity and quality of life. In Brazil, these results provided the bases for the Brazilian Health Regulatory Agency to approve the use of this medication for treatment of TTR cardiac amyloidosis at a dose of $80 \mathrm{mg} /$ day. ${ }^{17}$

To date, no published studies have evaluated the role of tafamidis specifically in patients with aortic stenosis; we may, however, infer that the medication remains effective, given that the pathophysiology of TTR cardiac amyloidosis does not differ in the context of aortic stenosis.

\section{Conclusion}

Aortic stenosis and TTR cardiac amyloidosis are 2 diseases that are very frequently associated, and the importance of their association has yet to be well

\section{References}

1. Otto CM, Prendergast B. Aortic-Valve Stenosis - From Patients at Risk to Severe Valve Obstruction. N Engl J Med. 2014 A;371(8):744-56. doi: 10.1056/ NEJMra1313875.

2. Simões MV, Fernandes F, Marcondes-Braga FG, Scheinberg P, Correia EB, Rohde LEP,etal. Posicionamento sobre Diagnósticoe Tratamento da AmiloidoseCardíaca -2021. Arq. Bras. Cardiol. 2021;117(3):561-98. doi: 10.36660/abc.20210718.

3. Tanskanen M, Peuralinna T, Polvikoski T, Notkola IL, Sulkava R, Hardy J, etal. Senile Systemic Amyloidosis Affects 25\% of the Very Aged and Associates with Genetic Variation in Alpha2-Macroglobulin and Tau: A Population-Based Autopsy Study. Ann Med. 2008;40(3):232-9. doi: 10.1080/07853890701842988.

4. Treibel TA, Fontana M, Gilbertson JA, Castelletti S, White SK, Scully PR, et al. Occult Transthyretin Cardiac Amyloid in Severe Calcific Aortic Stenosis: Prevalence and Prognosis in Patients Undergoing Surgical Aortic Valve Replacement. Circ Cardiovasc Imaging. 2016;9(8):e005066. doi: 10.1161/ CIRCIMAGING.116.005066. understood. Both diseases are prevalent in the elderly, and their prognosis is poor if they are not promptly identified and treated. Therapeutic decisions for these patients must be individualized and promptly discussed by the heart team for the patient's benefit. It is very important to identify which patients with aortic stenosis we should screen in order to avoid underdiagnosis of cardiac amyloidosis in this population and to offer the best treatment to all patients.

\section{Author contributions}

Conception and design of the research: Fernandes F, Kirschbaum M, Kalil Filho R, Tarasoutchi F. Acquisition of data: Kirschbaum M, Alencar Neto AC, Rosa VEE, Ramires FJA. Analysis and interpretation of the data: Fernandes F, Tarasoutchi F, Brito Junior FS, Brito Junior FS. Writing of the manuscript: Fernandes F, Kirschbaum M, Kalil Filho R, Tarasoutchi F, Alencar Neto AC. Critical revision of the manuscript for intellectual content: Fernandes $F$, Kirschbaum M, Kalil Filho R, Tarasoutchi F, Alencar Neto AC, Rosa VEE, Ramires FJA, Brito Junior FS, Abizaid AAC. Supervision / as the major investigador: Fernandes F, Kirschbaum M, Tarasoutchi F.

\section{Potential Conflict of Interest}

No potential conflict of interest relevant to this article was reported.

\section{Sources of Funding}

There were no external funding sources for this study.

\section{Study Association}

This study is not associated with any thesis or dissertation work.

\section{Ethics approval and consent to participate}

This article does not contain any studies with human participants or animals performed by any of the authors.
5. Rosenblum H, Masri A, Narotsky DL, Goldsmith J, Hamid N, Hahn RT, et al. Unveiling Outcomes in Coexisting Severe Aortic Stenosis and Transthyretin Cardiac Amyloidosis. Eur J Heart Fail. 2021;23(2):250-8. doi: 10.1002/ ejhf.1974.

6. Chacko L, Martone R, Bandera F, Lane T, Martinez-Naharro A, Boldrini M, et al. Echocardiographic Phenotype and Prognosis in Transthyretin Cardiac Amyloidosis. Eur Heart J. 2020;41(14):1439-47. doi: 10.1093/eurheartj/ ehz905.

7. Rapezzi C, Giannini F, Campo G. Aortic Stenosis, Transcatheter Aortic Valve Replacement and Transthyretin Cardiac Amyloidosis: Are We Progressively Unraveling the Tangle? Eur J Heart Fail. 2021;23(2):259-63. doi: 10.1002/ ejhf.2057.

8. Ando Y, Coelho T, Berk JL, Cruz MW, Ericzon BG, Ikeda S, et al. Guideline of Transthyretin-Related Hereditary Amyloidosis for Clinicians. Orphanet J Rare Dis. 2013;8:31. doi: 10.1186/1750-1172-8-31. 
9. Scully PR, Patel KP, Treibel TA, Thornton GD, Hughes RK, Chadalavada S, et al. Prevalence and Outcome of Dual Aortic Stenosis and Cardiac Amyloid Pathology in Patients Referred for Transcatheter Aortic Valve Implantation. Eur Heart J. 2020;41(29):2759-67. doi: 10.1093/eurheartj/ehaa170.

10. Peskó G, Jenei Z, Varga G, Apor A, Vágó H, Czibor S, et al. Coexistence of Aortic Valve Stenosis and Cardiac Amyloidosis: Echocardiographic and Clinical Significance. Cardiovasc Ultrasound. 2019;17(1):32. doi: 10.1186/s12947-019-0182-y.

11. Milandri A, Farioli A, Gagliardi C, Longhi S, Salvi F, Curti S, et al. Carpal Tunnel Syndrome in Cardiac Amyloidosis: Implications for Early Diagnosis and Prognostic Role Across the Spectrum of Aetiologies. Eur J Heart Fail. 2020;22(3):507-15. doi: 10.1002/ejhf.1742.

12. Nitsche C, Scully PR, Patel KP, Kammerlander AA, Koschutnik M, Dona C, et al. Prevalence and Outcomes of Concomitant Aortic Stenosis and Cardiac Amyloidosis. J Am Coll Cardiol. 2021;77(2):128-39. doi: 10.1016/j.jacc.2020.11.006.

13. Oda S, Takashio S, Nagamatsu S, Yamashita T, Uchimura R, Kidoh M, et al. Myocardial Extracellular Volume Quantification Using CT for the Identification of Occult Cardiac Amyloidosis in Patients with Severe Aortic Stenosis Referred for Transcatheter Aortic Valve Replacement. Amyloid. 2019;26(2):97-8. doi: 10.1080/13506129.2019.1597701.

14. Popma JJ, Deeb GM, Yakubov SJ, Mumtaz M, Gada H, O’Hair D, et al. Transcatheter Aortic-Valve Replacement with a Self-Expanding Valve in Low-Risk Patients. N Engl J Med. 2019;380(18):1706-15. doi: 10.1056/NEJMoa1816885.

15. Mack MJ, Leon MB, Thourani VH, Makkar R, Kodali SK, Russo M, et al. Transcatheter Aortic-Valve Replacement with a Balloon-Expandable Valve in Low-Risk Patients. N Engl J Med. 2019;380(18):1695-705. doi: 10.1056/ NEJMoa1814052.

16. Cavalcante JL, Rijal S, Abdelkarim I, Althouse AD, Sharbaugh MS, Fridman Y, et al. Cardiac Amyloidosis is Prevalent in Older Patients with Aortic Stenosis and Carries Worse Prognosis. J Cardiovasc Magn Reson. 2017;19(1):98. doi: 10.1186/s12968-017-0415-x.

17. Maurer MS, Schwartz JH, Gundapaneni B, Elliott PM, Merlini G, WaddingtonCruz M, et al. Tafamidis Treatment for Patients with Transthyretin Amyloid Cardiomyopathy. N Engl J Med. 2018;379(11):1007-16. doi: 10.1056/ NEJMoa1805689. 